\title{
Im Team gemeinsam zum Erfolg 1. Symposium für den ambulanten Bereich
}

Das „Unternehmen Transplantation“ kann nur gelingen, wenn alle Beteiligten in einem großen therapeutischen Regime zusammenarbeiten. Zum nephrologischen Team zählen selbstverständlich auch die niedergelassenen Nephrologen mit ihren Mitarbeitern. Im Mai haben wir daher zusammen mit Frau Prof. Petra Reinke und Herrn PD Andreas Pascher von der Charité Berlin das erste Transplantationssymposium für Mitarbeiter der Dialysen angeboten.

Die Transplantation ist ein wichtiges und etabliertes Verfahren für Patienten mit terminalem Nierenversagen. Die Ergebnisse nach Organtransplantation haben sich in den letzten zwei Jahrzehnten für alle Organbereiche wesentlich gebessert. Diese Erfolge sind auf optimierte immunsuppressive Schemata, chirurgische Techniken sowie verbesserte Aspekte der Patientenführung zurückzuführen und resultieren in steigenden Langzeitüberlebensraten und einer verbesserten Lebensqualität für den Einzelnen.

\section{Fortschritte und Probleme}

Ziel der Veranstaltung war es, die Fortschritte und immer noch existierenden Probleme der Organtransplantation an konkreten Patientenbeispielen nachvollziehbar zu machen. Eine Reihe von Patienten stellten ihre persönliche Sicht auf den Transplantationsverlauf vor und verstärkten somit das vermittelte Wissen emotional. Die wesentlichen Probleme in der Organtransplantation sind der Mangel an verfügbaren Organen, das Alter der Organspender und Empfänger und die Zunahme wiederholter bzw. kombinierter Transplantationen.

Lösungsansätze, die zur verbesserten Versorgung unserer Patienten beitragen sollen, sind zum einen der Ausbau der Lebendspendeaktivitäten, auch durch Akzeptanz von blutgruppenungleichen Spender-Empfänger-Konstellationen und die Einführung des sogenannten „old-forold“-Programms, in dem Organe von mindestens 65-jährigen Spendern auf ebenfalls ältere Empfänger übertragen werden. Zum anderen gilt es, kombinierte Organtransplantationen durchzuführen und komplex erkrankte Patienten zur Transplantation und Einführung neuer 
immunologischer Screeningverfahren zu akzeptieren, um Patienten mit komplexen Sensibilisierungen besser zu erkennen und über spezielle immunsuppressive Therapieregime erfolgreich zu transplantieren.

\section{Wissensvermittlung erlebbar machen}

Emotional bewegend war das Beispiel einer Patientin, die im Rahmen der ersten Transplantation aufgrund einer Nierenvenenthrombose das von der Mutter gespendete Transplantat verloren hatte. Die von der Patientin geschilderten Ängste, Zweifel, Schuldgefühle und Enttäuschungen gingen allen unter die Haut, obwohl diese wunderbar lebenslustige Frau nun im Rahmen einer zweiten Transplantation erfolgreich versorgt worden ist. Wie geht man mit einer solchen Situation als Arzt und Patient um? Wie klärt man im Vorfeld der Lebendspende auf, ohne die bereits emotional angespannte Situation überzustrapazieren? Wie nimmt man im Transplantationszentrum seine Verantwortung für die Spendernachsorge wahr? Wie vermittelt man trotz Misserfolg Vertrauen?

Vorgestellt wurde auch ein Lebendspendepärchen, bei dem der Ehemann trotz unterschiedlicher Blutgruppen als Spender akzeptiert werden konnte. Die Kombination aus speziellen neuen Medikamenten und neuartigen Blutwäscheverfahren ist ein wesentlicher Schlüssel zum Erfolg. Wie gestaltet man die immunologische Vorbereitung solcher Spender-Empfänger-Konstellationen und was muss generell bei immunologischen Hochrisikokonstellationen beachtet werden? Dr. Constanze Schönemann (Charité Berlin) erklärte in besonders anschaulicher Weise das aufwendige Antikörpersreening-Programm. Wie positioniert man sich bei der Akzeptanz zunehmend älterer Empfänger auf der Warteliste, die mit einer zum Teil kaum überschaubaren Liste von Diagnosen aufwarten? Ist es richtig und auch über die Jahre anhand der Langzeitfunktionsraten nachweisbar, dass „alte“ Organe einen guten Transplantationserfolg garantieren? Worauf muss in der Nachsorge noch geachtet werden?

\section{Fachübergreifende Kooperation notwendig}

Neben den auf der Hand liegenden Organfunktionen wird häufig ein großes „Organ“ - die Haut - sträflich missachtet. Einer unserer „old-for-old“Patienten, teilte uns Teilnehmern zwar freudestrahlend mit, dass er mit sehr guter Nierenfunktion seinen 35. Hochzeitstag feiern konnte, er schilderte aber auch die emotionale Belastung, die sich durch immer wiederkehrende Hauttumoren ergeben. Daran zu denken, Patienten, Schwestern und Ärzte zu schulen und die Kooperation mit erfahrenen Hautärzten sind die Basis für eine gute Frühdiagnose, optimale Therapie und wenig „verstümmelnde" Befunde.

Für alle sehr bewegend war das Schicksal eines jungen Mannes, der aufgrund einer immunologischen Darmerkrankung eine Vielzahl von Darmoperationen über sich ergehen lassen musste. Letztlich endete dieser medizinische Exodus mit einem Kurzdarmsyndrom, einer sogenannten sekundären Oxalose und dem chronischen Nierenversagen. Das erste Transplantat war innerhalb recht kurzer Zeit wieder durch die Oxalatablagerung geschädigt und da keine Gefäßanschlussmöglichkeit für die Dialyse und bei den häufigen Bauchoperationen auch keine Möglichkeit zur Bauchfelldialyse bestand, wurde er vor vier Jahren im Rahmen der höchsten Transplantationsdringlichkeit zum zweiten Mal erfolgreich transplantiert. Mit welchem ausgefeilten Ernährungsregime der Patient seither in einem erstklassigen Ernährungszustand mit guter Transplantatfunktion als selbstständig tätiger Medizinjournalist sein Leben gestaltet, zeigt einmal mehr, was im Rahmen der Organtransplantation an Lebensgewinn möglich ist. Es herrschte fast andächtige Stille und auch die anderen Patienten, die alle ihr „Päckchen“ zu tragen haben/hatten, waren beeindruckt. Das einhellige Resümee der Veranstaltung sowohl bei Patienten als auch Teilnehmern war, „macht so eine Veranstaltung wieder, macht Wissensvermittlung erlebbar".

Petra Hecker, 1.Vorsitzende AKTX-Pflege e. V. 